\title{
Retraction Note: Dringliche oder notfallmäßige Koronarrevaskularisation unter Verwendung beider Arteria Thoracica interna nach vorheriger Thrombozytenaggregationshemmung mit Clopidogrel
}

\author{
F. Schmidtler $\cdot$ B. Gansera $\cdot$ K. Spiliopoulos $\cdot$
}

I. Angelis $\cdot$ P. Neumaier-Prauser $\cdot$ B. M. Kemkes

Published online: 29 November 2012

(c) Springer-Verlag Berlin Heidelberg 2012

Retraction to: Z Kardiol (2004) 93:679-685

DOI 10.1007/s00392-004-0115-z

This article has been retracted as it represents a substantial duplication of the article: B. Gansera, F. Schmidtler, K. Spiliopoulos, I. Angelis, P. Neumaier-Prauser, B.M. Kemkes: Urgent or emergent coronary revascularization using bilateral internal thoracic artery after previous clopidogrel antiplatelet therapy. Thorac Cardiov Surg (2003) 51:185-189.

The online version of the original article can be found under doi: $10.1007 / \mathrm{s} 00392-004-0115-\mathrm{z}$.

F. Schmidtler $(\bowtie) \cdot$ B. Gansera · K. Spiliopoulos · I. Angelis ·

P. Neumaier-Prauser · B. M. Kemkes

Krankenhaus München-Bogenhausen, Abt. für

Kardiovaskularchirurgie, Englschalkingerstr. 77,

81925 München, Germany 